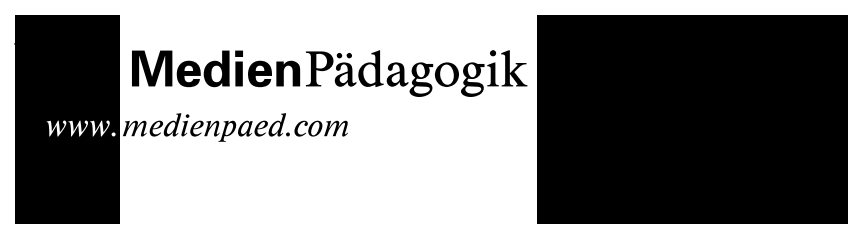

Johannes Busse, Benno Volk, Christiane Schiersmann

22.7.2003

\section{Content Management von Leittexten mit XML Topic Maps}

\section{Einleitung}

Der Umgang mit internet-basierten Informations- und Kommunikationstechnologien (IuK-Technologien) stellt in der heutigen Zeit eine Schlüsselkompetenz für Studierende sämtlicher Fachrichtungen dar. Von den Universitäten als berufsqualifizierende Bildungsinstitutionen wird erwartet, angemessen auf diese Entwicklung zu reagieren. «Die Implementierung der neuen Informations- und Kommunikationstechnologien in den Lehr- und Lernprozess wird als unerlässliche Voraussetzung für die Hochschulentwicklung, für inhaltliche und strukturelle Reformen der Hochschulausbildung gewertet» (BMBF 2002). Es bedarf daher geeigneter Strategien, wie dieses komplexe Wissen in das jeweilige Hochschulstudium integriert und nachhaltig vermittelt werden kann.

Das seit 2001 am Erziehungswissenschaftlichen Seminar der Universität Heidelberg etablierte Entwicklungsprojekt «Studbene» bietet hierzu einen konzeptionellen Ansatz. Auf Grundlage eines handlungsorientierten Curriculums und teilnehmeraktivierender Vermittlungsformen bildet das Projekt in einem Zeitraum von zwei Semestern Studierende aus geistes- und sozialwissenschaftlichen Fächern zu «Lernberater/innen für Neue Medien» aus. Das Ziel des Projekts besteht in der Vermittlung einer praxistauglichen Medienkompetenz sowie einer themenzentrierten Beratungskompetenz im Sinne der «lösungsorientierten Kurzberatung» (vgl. Wolters 2000; Bamberger 2001).

Die studentischen Lernberater/innen verfügen am Ende der einjährigen Ausbildung über Kompetenzen bezüglich methodisch-didaktischer und organisatorisch-technischer Aspekte bei der Entwicklung und Umsetzung mediengestützter Lernstrategien. Sie fungieren als Multiplikator/innen, die ihre erworbenen Kompetenzen entsprechend der Ausbildung in «Studbene» an andere Personen weiter vermitteln. Weil sie in erster Linie Studierende und mittelbar auch Dozent/innen beraten, fördern sie eine «Hochschulentwicklung von unten». Sowohl die Ausbildung als auch die Tätigkeit der Lernberater/innen findet an einer klassischen Präsenzhochschule statt, deren Charakter erhalten bleiben soll. Die hierzu geeigneten hybriden Lehrund Lernarrangements unterscheiden sich deutlich von anderen Vorstellungen zur «Virtualisierung» der Hochschullehre.

Der Lösungsansatz von «Studbene» für diese komplexe Herausforderung beruht auf drei strategischen Entscheidungen:

(1) Ein wichtiges didaktisches Fundament bildet die «Leittextmethode». Angepasst an das mediengestützte Lehr-/Lernkonzept und den universitären Kontext, fördert diese Methodik das selbstentdeckende Lernen in Einzel- sowie Gruppenarbeiten. Sie ermöglicht zudem eine Vermittlung von pädagogisch-didaktischer Grundkompetenz, die für die Beratungstätigkeit der Teilnehmenden von Bedeutung ist.

(2) Inhaltlich orientiert sich die Lehre von «Studbene» soweit als möglich an open source-Standards, die eine medien-neutrale und hersteller-unabhängige Medienkompetenz garantieren. Als wichtiger curricularer Bestandteil wurde hierfür eine Einführung in die XML-Technologie am Beispiel der Webseitenerstellung mit XHTML und dem Content-Management mittels einer XML Topic Map ausgewählt.

(3) Organisatorisch wird das Multiplikatorenkonzept durch die Initiierung eines Netzwerks der Lernberater/innen über die Zeit der eigentlichen Ausbildung hinaus unterstützt. Entsprechend dem Konzept von «Studbene» wird dabei dem Aspekt des sozialen Lernens eine fundamentale Bedeutung beigemessen.

Diese drei strategischen Entscheidungen und die damit verbundenen Lerninhalte sind eng miteinander verzahnt: Die Leittextmethode wird entsprechend dem XML-basierten XHTML-Standard in Form von online Publikationen umgesetzt. Dieser Textbestand wird mit Hilfe einer Topic Map strukturiert, die wiederum eine praktische Anwendung von XML darstellt. Die Topic Map ermöglicht den Lernberater/innen zudem, netzbasiert auf die Inhalte von «Studbene» nicht nur «lesend» zugreifen, sondern auch «schreibend» eigene Inhalte in dieser Form in den Textbestand integrieren zu können. Auf diesem Wege wird ein Austausch von Unterlagen für die Beratungstätigkeit im Sinne einer «Community of Practice» realisiert. 
Nach Überwindung der Anlaufwiderstände in der Pilotphase haben die Studierenden nach eigenen Angaben diese Lehrinhalte als grosse Chance für ihre berufliche Zukunft erkannt; sie zeigten durchweg ein aussergewöhnlich grosses Engagement und eine Begeisterung für die angesprochenen Themengebiete. Dies und die interdisziplinäre Zusammensetzung der Teilnehmergruppe führten zu einem regen sozialen Austausch unter Teilnehmern und Dozenten. Die überwiegende Mehrzahl der Absolventen/innen der Pilotphase arbeitet derzeit als Lernberater/innen und setzt ihre Projektideen in unterschiedlichen Instituten der Universität Heidelberg um.

\section{Die Leittextmethode}

In einem ersten Durchlauf der Ausbildung mit 12 Teilnehmenden wurde deutlich, dass sich die Lernziele aufgrund der hohen Komplexität der Lehrinhalte und dem grossen Stoffumfang in einem begrenzten zeitlichen Rahmen (Norm je Semester: 120 Stunden Präsenzzeit, 240 Stunden Selbststudien- und Kleingruppenarbeitszeit) nur durch ein Lernarrangement erreichen lassen, bei dem eine hohe Teilnehmeraktivierung mit selbstgesteuerten und kooperativen Gruppenlernprozessen erreicht wird.

In dieser Pilotphase wurde die Leittextmethode weiter entwickelt. Dieses Konzept aus den 70er Jahren für eine situations- und problemorientierte Aneignung von praktischen Handlungskompetenzen im metallverarbeitenden Gewerbe schien auch für die Studierenden in «Studbene» als Form des «explorativen Lernens» geeignet.

In der klassischen Leittextmethode sollen die Lernenden nach einer kurzen Einführung in das jeweilige Thema sowie durch spezielle weitergehende Fragestellungen angeleitet werden, notwendige Kenntnisse, Fähigkeiten und Fertigkeiten in Form einer Projektarbeit selbständig anzueignen. Diese Form des Lernens unterstützt somit die Aneignung von Handlungskompetenz, wie auch eine Selbstorganisation des Lernprozesses. Das Rollenverständnis der Ausbilder/innen wandelt sich dementsprechend von einer unmittelbaren Vermittlerrolle hin zu einer Begleiter- und Beraterrolle. Die Leittextmethode wurde später u. A. von Greif (1996) weiter ausgearbeitet und für die universitäre Lehre fruchtbar gemacht. Ausgehend von diesem Stand wird die Methode für ein aktives Lernen von Fach-, Methoden- und Sozialkompetenz eingesetzt. (vgl. Golle/Hellermann 2001) Auf der Basis des durch o. a. Lehr- und Lernkontexts werden in «Studbene» drei standardisierte Texttypen ausdifferenziert:
- Informationstexte stellen Inhalte sachsystematisch dar und vermitteln ein notwendiges Grundwissen zu den Themenbereichen. Wir entwickeln diese Informationstexte selbst, verweisen jedoch in hohem Mass auch auf ausgesuchte Internet-Ressourcen.

- Studbene-Leittexte (SLT) enthalten Vorgehensvorschläge, Leifragen und/oder Übungen, um sich auf der Basis von Informationstexten und den weiteren Ressourcen spezifische Handlungskompetenzen aneignen zu können. Die Leittexte sind ergebnisorientiert und haben durch den stufenartigen Aufbau des Lernprozesses auch eine motivierende Funktion zum Gelingen des selbstorganisierten Lernens.

- Session-Planungstexte (SPT) sind mikrodidaktische Modelle für Unterrichtseinheiten von 90 Minuten («Sessions») im Sinne von verschriftlichten, medial «konservierten» Sitzungplanungen und -abläufen. Sie sind zielorientiert aufgebaut, indem einzelne Lernergebnisse und die am Ende angestrebten Kompetenzen vorab benannt werden.

Die in einer Session eingesetzten Informations-, Leit- und Planungstexte stehen in engem Bezug zueinander. Die entstehenden Pakete aus drei aufeinander aufbauenden Textformen wiederum machen in ihrer Gesamtheit den «Content» des Wissensnetzwerks aus.

Während in den Informationstexten je nach Zielsetzung unterschiedlich viel Grundwissen zu einem Themengebiet vorgegeben werden kann, leisten die Leittexte eine handlungsorientierte Didaktisierung von Inhalten. Die Planungstexte wiederum fügen die einzelnen Materialien zu einem prozesshaften Lernberatungskonzept zusammen. Da es sich bei «Studbene»um eine Ausbildung von «Multiplikatoren» handelt, die ihr erwobenes Wissen und die methodisch-didaktischen Erfahrungen aus der Ausbildung in ihrer späteren Tätigkeit als Lernberater/innen weitergeben, mussten die angebotenen Lernmaterialien so konzipiert werden, dass sie sowohl als Grundlage für diese Formen des Lernens, als auch zur medien-neutralen, XML-konformen Veröffentlichung im Internet geeignet sind. Die Verbindung von netzbasierten und standardisierten Planungstexten, Informationstexten und Leittexten in der Topic Map stellt eine mediale Abbildungsform pädagogisch-didaktischer Praxis dar.

Indem die Leittextmethode in dieser Form zum Lehrgegenstand der Ausbildung gemacht wird, geht es nicht alleine um einen medialen, sondern auch um einen pädagogisch-didaktischen Anspruch. Lernprozesse der Teilnehmenden finden hierbei zum einen durch die Verwendung der 
Leittexte während der Erstausbildung und durch das eigene Schreiben von Leittexten als Form der Ergebnissicherung statt.

Die Zielrichtung hierbei ist der Aufbau eines Wissensnetzwerks zur Unterstützung der Beratungstätigkeit: Leittexte und andere Materialien aus der Ausbildung sollen auch zur Lernberatung eingesetzt werden und durch den gemeinsamen Austausch einen Kompetenz-Transfer und die Bildung eines Netzwerks (i. S. einer «Community of Practice») fördern.

Die Inhalte dienen somit der «Studbene»-Ausbildung und den ausgebildeten Lernberater/innen als Lehrmaterialien. Eine strukturierte Organisation und die nutzergerechte Darstellung sind daher wichtige Kriterien für die nachhaltige Wiederwendungsmöglichkeit. Hierzu wurde mit der Entwicklung einer «Studbene Topic Map» (STM) eine innovative, ausbaufähige und auf vielfältige Anwendungsgebiete übertragbare Lösung gefunden.

\section{Leittext-Management mit XML Topic Maps}

Im vorangehenden Abschnitt haben wurde gezeigt, dass Leittexte nicht nur intra-textuell eine hohe formale Strukturierung aufweisen, sondern auch inter-textuell eng aufeinander bezogen sind. Im Laufe des Aufbaus des Textbestandes wurde zunehmend deutlich, dass insbesondere die Struktur «Zwischen» den einzelnen Texten erziehungswissenschaftlichen Content implizit wiederspiegeln. Um diesen Content in hybriden Lernarrangements für Lehren und Lernen handhabbar und fruchtbar machen zu können, müssen Herausforderungen bezüglich des technischen Managements sowie der «sozialen» Semantik von Querverweisen gelöst werden.

\section{Herausforderung 1: Querverweis-Management}

Die verschiedenen (Informations-, Leit- und Planungs-) Texte müssen nicht nur technisch zugänglich gemacht, sondern auch recherchierbar und dazu sachlogisch erschlossen werden. Schon alleine die intertextuelle LinkIntegrität aufrecht zu erhalten ist nicht ganz einfach; wirklich komplex wird die sachlogische Erschliessung, wenn (1) in einer grossen Datei viele kleine (Mikro-)Sinneinheiten enthalten sind, auf die auch separat Bezug genommen werden soll (z. B. Info- und Aufgaben-Blöcke, Darstellungen etc.), und gleichzeitig (2) grössere (Makro-)Sinneinheiten sich erst aus dem Zusammenfügen einzelner Dateien ergeben (wie es z. B. bei einem kollaborativ erstellten Aufsatz der Fall sein kann). Falls an diesem Prozess der Content-Erstellung mehr als eine Person - etwa wie in «Studbene» mehrere Dozent/innen und ein gutes Dutzend Lernberater/innen - beteiligt werden sollen, ist ein gut überlegtes Content Management unerlässlich.

Wir versuchen uns dieser Herausforderung zu stellen, indem wir die intertextuellen Querbezüge auf Basis des Standards XML Topic Maps (XTM) modellieren. Das in jeder Topic-Map enthaltene Produkt, das für den indizierten Gegenstand mit einer spezifischen kategorialen Begrifflichkeit ( Ontologie») entwickelt wird, nennen wir Studbene Topic Map (STM).

Eine STM lässt sich als semantisches Netzwerk verstehen, in dem «Topics» (Wissensinhalte) miteinander «assoziiert (semantisch verbunden) sind, indem sie in paarweise zueinander passende Rollen schlüpfen. Beispiel: In der Assoziation Schriften spielt der Topic JB die Rolle Autor. Er steht damit in Relation zum Topic mewiss03, der die Rolle Aufsatz spielt. Dieser Tatbestand kann in XML etwa wie folgt dargestellt werden:

\section{$<!--$ Wir assoziieren} $-->$

$<$ !-- innerhalb (einer Instanz) der Association «Schriften» --> $<$ Assoc $>$ Schriften

\section{$<!--\ldots$ den Topic JB in der Rolle Autor}

$<$ Autor>JohannesBusse</Autor>

<!-- ... mit dem Topic mewiss03 in der Rolle Aufsatz -->

$<$ Aufsatz>mewiss $03</$ Aufsatz>

\section{$</$ Assoc $>$}

Da es ohnehin zum «Studbene»-Curriculum gehört, Texte auch in (einfachem) XHTML codieren zu können, ist der Schritt zum Topicmapping nicht mehr gross: Die Studierenden erlernen diese Grundlagenwissen bezüglich XML und werden ermutigt, solche XML-Fragmente direkt in selbst erstellte XHTML-Dateien einzufügen. Für den Webserver wurden kleine XSLT-Stylesheets geschrieben, die solche Fragmente auswerten und zu einer integrierten XTM zusammenfügen können - und zwar (1) in einem einzigen Arbeitsgang (2) auf Grundlage von komplexen Sammlungen von Texten (3) insbesondere auch von Studierenden und (4) unabhängig davon, wo diese Texte gehostet werden. Die einzige Bedingung ist, dass die zu indizierenden Texte über eine URL erreichbar sind und offenen Standards genügen. Mit geringem technischem Aufwand entsteht so ein nutzer- 
freundliches, in vorhandene Texte einfach eingebettetes, am Prinzip des literate programming orientiertes Codierungssystem für kollaboratives Topicmapping.

Im Prototyp ist dieser Ansatz für das Content Management des Projekts «Studbene» verwirklicht, wobei derzeit vorwiegend inter-textuelle Assoziationen erfasst sind. Weitergehend wird daran gearbeitet, auch die Binnenstrukturen von den definierten Texttypen (wie etwa SPT und SLT) und damit intra-textuelle Strukturen in der XTM zu codieren und zu erfassen.

Herausforderung 2: Querverweis-Semantik

Integrität von Verweisen («Links») technisch aufrecht zu erhalten ist zwar eine notwendige, jedoch keine hinreichende Bedingung für eine angemessene Lösung. Zusätzlich muss eine soziale Übereinkunft darüber erzielt werden, welche der vielen denkbaren Querbezüge zwischen den Texten als «sinnvoll» gelten sollen. Eine bekannte Problematik, die sich beim gemeinsamen Erstellen einer solchen Struktur stellt, ist die Frage nach der Vermittlung der vereinbarten Normen sowohl für die Nutzer/innen wie auch für Autor/innen.

Bei der kollaborativen Erstellung einer XTM muss insbesondere eine Übereinkunft darüber erzielt werden, welche Typen von Topics sich auf welche Art und Weise assoziieren lassen, und welche typischen Rollen-Paare dabei eingenommen werden können. (In der Sprache der Topic Maps wird diese Übereinkunft eine Ontologie genannt). Um eine solche Übereinkunft zu kommunizieren haben wir eine grafische Notation entwickelt:

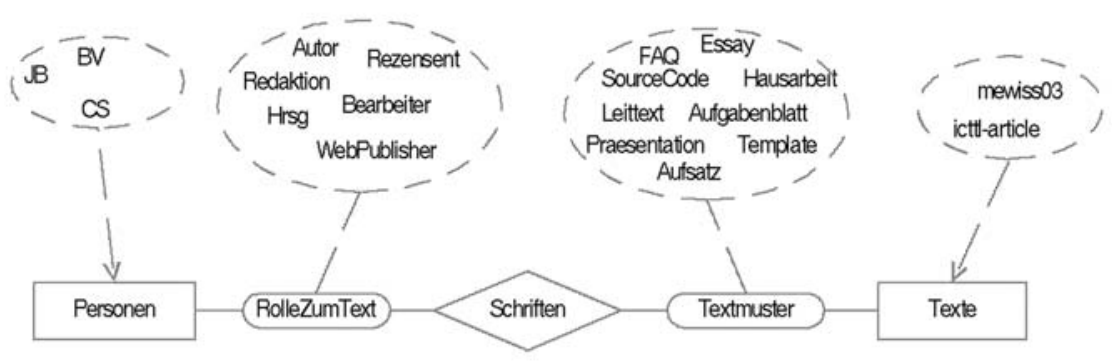

Diese Grafik kann folgendermassen gelesen werden: (1) Es gibt einen Topic «mewiss03» (in «Studbene» ein eindeutiger Identifikator des vorliegenden Aufsatzes) vom Typ «Texte». (2) Jeder Topic vom Typ
«Personen» kann eine der Rollen «Autor», «Hrsg.» etc., jeder Topic vom Typ «Texte» kann eine der Rollen «Leittext», «Aufsatz» etc. einnehmen -und zwar dann und nur dann, wenn sie miteinander in der Assoziation «Schriften» assoziiert sind.

Wir führten Details an dieser Stelle aus verschiedenen Gründen aus. Zum einen wollen wir einen Eindruck davon vermitteln, welche auf formale Strukturen bezogenen Bildungsinvestitionen wir im Projekt «Studbene» von unseren Lernberater/innen verlangen. Vor allem aber bildet der beschriebene Formalismus die Basis, um im folgenden Abschnitt konkret die erziehungswissenschaftliche Relevanz von formalen Strukturen nicht nur für das Lehren, sondern insbesondere für das Lernen deutlich zu machen.

Didaktische Qualität: Lernen durch hoch strukturiertes Schreiben

Ein Formalismus, wie er für eine XML Topic Map notwendig ist, lässt sich nicht lediglich «rezeptiv», d. h. im Lesezugriff z. B. zur leichteren Navigation in komplexen Wissensbeständen, sondern auch «aktiv», im Schreibzugriff als Framework für die Externalisierung von Wissen nutzen. Einen Wissensbestand zunächst in viele kleine Teile aufzugliedern und dann z. B. mittels einer XTM zu einem Netz von semantisch gehaltvollen Assoziationen zusammenzufügen, ist eine anspruchsvolle Form des Authoring. Was aus technischer Sicht zunächst «nur» ein Format, eine Norm oder ein Formalismus zu sein scheint, entpuppt sich in der Hand der Lernenden als Werkzeug zum Aufbau einer Knowledge Map als hoch strukturierte Wissensexternalisierung.

In der experimentellen psychologischen Forschung ist der Einsatz von knowledge maps insbesondere für die ergebnissichernde Rekonstruktion von Wissenstrukturen im Anschluss, an auf Lernen ausgerichtete Treatments seit langem wohlbekannt (z. B. Cimolino and Kay 2002). Wir versuchen mit unserem Ansatz, solche Techniken auch schon in frühen Phasen des Lernzyklus fruchtbar einzusetzen. Gemäss den Prinzipien «Lernen durch Lehren» und «Lernen durch Schreiben» glauben wir einen Formalismus wie den XTM-Standard als formale Schrift und als solche, als ein wichtiges Werkzeug für Lernende einsetzen zu können.

Damit dies klappt müssen allerdings einige Voraussetzungen erfüllt sein. (1) Die Lernenden müssen dazu bereit sein, sich auch auf ausgewählte Wissensbestände aus den Strukturwissenschaften (wie z. B. der Informatik) einzulassen. (2) Die Lernarrangements müssen so beschaffen sein, dass gleichzeitig hoch strukturierte und von Studierenden erstellte Texte in der 
Lehre zur Verwendung kommen können. Beides ist Kennzeichen unserer Didaktik in «Studbene».

\section{Zusammenfassung}

Das didaktische Konzept von «Studbene» integriert drei Aspekte: (1) Die im beruflichen Bildungswesen entwickelte Leittextmethode wurde auf hybride Lernarrangements im tertiären Bildungsbereich transferiert. (2) XML und XML Topic Maps wurden als genuiner curricularer Inhalt der Ausbildung definiert, der auch im Projekt selbst breit zur Anwendung kommt. (3) Zur Unterstützung des Einsatzes der Lernberater/-innen und der Fortschreibung der Ausbildung wurde auf Basis der Aspekte 1 und 2 ein sozio-technisches Netzwerk aufgebaut.

Wir glauben mit dieser Integration dort Neuland zu betreten, wo wir eine Darstellungsmethode aus den Strukturwissenschaften als Medium Formale Sprache interpretieren und für die Modellierung von erziehungswissenschaftlichem Content nutzen - nämlich von Content, der zwar «zwischen» einzelnen (hoch strukturierten) Texten implizit enthalten ist, von den Texten selbst jedoch kaum repräsentiert wird. Dieses didaktische Konzept öffnet die Hochschullehre auch gemäss dem Ideal einer interdisziplinäreren Ausrichtung, bereichert das erziehungswissenschaftliche Studium methodisch wie curricular und trägt so zu einer praxisorientierten, berufsqualifizierenden Ausbildung bei.

\section{Literatur}

International Labor Office (Hrsg.). Approaches to learning at work: Box 3: The Leittext Method. Geneva (Swiss), 1998.

〈http://www-ilo-mirror.cornell.edu/public/english/employment/skills/ recomm/publ/019.htm\#Leittext>

Aroyo, L; Dicheva, D. (Hrsg.). Workshop on Concepts and Ontologies in Web-based Educational Systems: ICCE 2002, International Conference on Computers in Education, 3-6 December 2002, Auckland, New Zealand. 〈http://wwwis.win.tue.nl/ laroyo/ICCE2002_Workshop/procWorkshop-ICCE2002.pdf $>$ (2002).

Ballstaedt, Steffen-Peter; Will, Hermann (Hrsg.). Lerntexte und Teilnehmerunterlagen. Weinheim, 1991.

BMBF; Middendorff, Elke (Hrsg.). Computernutzung und Neue Medien im Studium. Bonn, 2002.
Cimolino, Laurent; Kay, Judy. Verified Concept Mapping for Eliciting Conceptual Understanding. 2002.

Geiger, Robert; Seubert, Peter. Berufspädagogisches Wörterbuch DeutschEnglisch. München, 2003.

〈http://www.lrz-muenchen.de/ geigerpublikationen/dict/dict.htm〉

Golle, Karen; Hellermann, Klaus (Hrsg.). Leittextgestütztes Lehren und Lernen in der Hochschule. Bochum, 2001.

Greenberg, Jane. Semantic Web Construction: An Inquiry of Authors' Views on Collaborative Metadata Generation.

〈http://www.bncf.net/dc2002/program/ft/paper5.pdf〉 (2002).

Greif, Siegfried (Hrsg.). Handbuch Selbstorganisiertes Lernen. Göttingen, 1996a.

Pepper, Steve. The TAO of Topic Maps.

〈http://www.ontopia.net/topicmaps/materials/tao.html〉 (2000).

Schweizer, Gerd (Hrsg.). Methodenkompetenz lehren und lernen: Beiträge zur Methodendidaktik in Arbeitslehre, Wirtschaftslehre und Wirtschaftsgeographie. Dettelbach: Verlag J.H. Röll, 2001.

Tannenbaum, Adrienne. Metadata solutions: using metamodels, repositories, XML, and enterprise portals to generate information on demand. Boston, London: Addison-Wesley, 2001.

Quelle und ggf. aktualisierte Fassung des vorliegenden Aufsatzes siehe http://www.studbene.de/stm/mewiss03.html 